\title{
SUATU KAJIAN TITIK TETAP PEMETAAN $k$-PSEUDONONSPREADING SEJATI DI RUANG HILBERT
}

\author{
DESI RAHMADANI \\ Program Studi Matematika, \\ Fakultas Matematika dan Ilmu Pengetahuan Alam, Universitas Andalas, \\ Kampus UNAND Limau Manis Padang, Indonesia, \\ desi.rahmadani39@gmail.com
}

\begin{abstract}
Let $C$ be a subset of a real Hilbert space $H$. Let $T: H \rightarrow H$ be a strictly $k$-pseudononspreading mapping with a nonempty fixed point set. Let $\omega \in[k, 1)$ be fixed. Let $\left\{T_{i}\right\}_{i=1}^{N}$ be $N k_{i}$-strictly pseudononspreading mappings. In this paper, we study the relationship between of a fixed point set of a $k$-strictly pseudononspreading mapping and other forms of certain combinations of some $k$-strictly pseudononspreading mappings in Hilbert space.
\end{abstract}

Kata Kunci: $k$-strictly pseudononspreading mapping, Hilbert space.

\section{Pendahuluan}

Teori ruang Hilbert pertama kali diperkenalkan oleh David Hilbert pada tahun 1909. Kajian ini mengawali penelitian dalam bidang analisis fungsional di ruang berdimensi tak hingga, yang kemudian lebih dikenal dengan sebutan ruang Hilbert. Selanjutnya, John Von Naumann merumuskan sifat-sifat teori ruang Hilbert dan mengembangkan teori baru dari operator-operator dalam ruang Hilbert.

Dalam [7], Osilike dan Isiogugu memperkenalkan suatu pemetaan baru yang disebut dengan pemetaan $k$-pseudononspreading sejati. Misalkan $T: C \rightarrow H, C$ adalah suatu himpunan bagian tak kosong, tertutup dan konveks dari ruang Hilbert riil $H$. Pemetaan $T$ dikatakan pemetaan $k$-pseudononspreading sejati jika terdapat $k \in[0,1)$ sedemikian sehingga

$$
\begin{aligned}
\|T x-T y\|^{2} \leq & \|x-y\|^{2}+k\|x-T x-(y-T y)\|^{2}+2\langle x-T x, y-T y\rangle, \\
& \forall x, y \in C .
\end{aligned}
$$

Sedangkan $T$ dikatakan pemetaan $k$-pseudocontraction sejati jika terdapat $k \in[0,1)$ sedemikian sehingga

$$
\|T x-T y\|^{2} \leq\|x-y\|^{2}+k\|x-T x-(y-T y)\|^{2}, \forall x, y \in C
$$

Dalam makalah ini akan dikaji hubungan himpunan titik tetap dari suatu pemetaan $k_{i}$-pseudononspreading sejati $\left\{T_{i}\right\}_{i=1}^{N}$ dengan bentuk-bentuk kombinasi tertentu dari beberapa pemetaan $k$-pseudononspreading sejati di ruang Hilbert yang berdasarkan pada dua fakta berikut, sebagaimana yang ditulis dalam [7]: 
Titik Tetap Pemetaan k-pseudononspreading di Ruang Hilbert 53

(1) Terdapat titik tetap dan hubungan titik tetap dari pemetaan $k_{i^{-}}$ pseudocontraction sejati $\left\{T_{i}\right\}_{i=1}^{N}$ dengan bentuk-bentuk kombinasi tertentu dari beberapa pemetaan tersebut di ruang Hilbert [1].

(2) Terdapat titik tetap pada pemetaan $k$-pseudononspreading sejati dan menunjukkan bahwa titik tetap ini merupakan solusi untuk suatu masalah optimasi [2].

\section{Kajian Titik Tetap Pemetaan $k$-pseudononspreading Sejati di Ruang Hilbert}

Sebelum mengkaji bukti dari hubungan himpunan titik tetap dari suatu pemetaan $k_{i}$-k-pseudononspreading sejati $\left\{T_{i}\right\}_{i=1}^{N}$ dengan bentuk-bentuk kombinasi tertentu dari beberapa pemetaan $k$-pseudononspreading sejati di ruang Hilbert, akan dibuktikan beberapa lema berikut.

Lema 2.1. [3] Misalkan $H$ adalah ruang Hilbert riil. Maka pernyataan berikut berlaku:

(1) $\|t x+(1-t) y\|^{2}=t\|x\|^{2}+(1-t)\|y\|^{2}-t(1-t)\|x-y\|^{2}, \forall x, y \in H, \forall t \in[0,1]$,

(2) $\|x-y\|^{2} \leq\|x\|^{2}+2\langle y, x+y\rangle, \forall x, y \in H$.

\section{Bukti.}

(1) $\|t x+(1-t) y\|^{2}=t\|x\|^{2}+(1-t)\|y\|^{2}-t(1-t)\|x-y\|^{2}, \forall x, y \in H, \forall t \in[0,1]$.

$$
\begin{aligned}
\|t x+(1-t) y\|^{2} & =\langle t x+(1-t) y, t x+(1-t) y\rangle \\
& =\langle t x, t x\rangle+2\langle t x,(1-t) y\rangle+\langle(1-t) y,(1-t) y\rangle \\
& =t^{2}\langle x, x\rangle+2\langle t x,(y-t y)\rangle+\langle y-t y, y-t y\rangle \\
& =t^{2}\langle x, x\rangle+2(\langle t x, y\rangle-\langle t x, t y\rangle)+\langle y, y\rangle-2 t\langle y, y\rangle+\langle t y, t y\rangle \\
& =t^{2}\langle x, x\rangle+2 t\langle x, y\rangle-2 t^{2}\langle x, y\rangle+\langle y, y\rangle-2 t\langle y, y\rangle+\langle t y, t y\rangle \\
& =t\langle x, x\rangle+\langle y, y\rangle-t\langle y, y\rangle-\left(t-t^{2}\right)(\langle x, x\rangle+2\langle x, y\rangle+\langle y, y\rangle) . \\
& =t\langle x, x\rangle+\langle y, y\rangle-t\langle y, y\rangle-\left(t-t^{2}\right)\langle x-y, x-y\rangle \\
& =t\langle x, x\rangle+(1-t)\langle y, y\rangle-t(1-t)\langle x-y, x-y\rangle \\
& =t\|x\|^{2}+(1-t)\|y\|^{2}-t(1-t)\|x-y\|^{2} .
\end{aligned}
$$

(2) $\|x-y\|^{2} \leq\|x\|^{2}+2\langle y, x+y\rangle, \forall x, y \in H$.

$$
\begin{aligned}
\|x-y\|^{2}=\langle x-y, x-y\rangle=\langle x, x\rangle-2\langle x, y\rangle+\langle y, y\rangle & \leq\langle x, x\rangle+2\langle x, y\rangle+2\langle y, y\rangle, \\
& =\langle x, x\rangle+2\langle y, x+y\rangle, \\
& =\|x\|^{2}+2\langle y, x+y\rangle,
\end{aligned}
$$

sehingga, $\|x-y\|^{2} \leq\|x\|^{2}+2\langle y, x+y\rangle, \forall x, y \in H$.

Berikut adalah lema yang menjelaskan hubungan dari suatu pemetaaan $k$ pseudononspreading dengan suatu bentuk pemetaan $k$-pseudononspreading yang didefinisikan di ruang Hilbert. 
Lema 2.2. [3] Misalkan $C$ merupakan himpunan bagian dari ruang Hilbert riil $H$, dan misalkan $T: H \rightarrow H$ merupakan suatu pemetaan $k$-pseudononspreading sejati dengan suatu himpunan titik tetap tak kosong. Misalkan $\omega \in[k, 1)$ ditetapkan dan didefinisikan $T_{\omega}: C \rightarrow C$ dengan

$$
T_{\omega} x=(1-\omega) T x+\omega x, \forall x \in C .
$$

$\operatorname{maka} F\left(T_{\omega}\right)=F(T)$.

Bukti. Misalkan $T_{\omega} x=(1-\omega) T x+\omega x$, atau dapat ditulis $x-T_{\omega} x=(1-\omega)(x-T x)$, $\forall x \in C$.

(i) Akan dibuktikan $F\left(T_{\omega}\right) \subset F(T)$. Misalkan $x \in F\left(T_{\omega}\right)$ dengan $x=T_{\omega} x$. Dari persamaan (2.1) diperoleh

$$
\begin{aligned}
T_{\omega} x & =(1-\omega) T x+\omega x \\
x & =(1-\omega) T x+\omega x \\
x & =(1-\omega) T x+\omega x \\
x-\omega x & =(1-\omega) T x \\
(1-\omega) x & =(1-\omega) T x .
\end{aligned}
$$

Karena $(1-\omega) \neq 0$, maka $x=T x$. Sehingga terbukti $x \in F(T)$.

(ii) Akan dibuktikan $F(T) \subseteq F\left(T_{\omega}\right)$. Misalkan $x \in F(T)$ dengan $x=T x$. Dari persamaan (2.1) diperoleh

$$
\begin{aligned}
& T_{\omega} x=(1-\omega) T x+\omega x \\
& T_{\omega} x=(1-\omega) x+\omega x \\
& T_{\omega} x=x-\omega x+\omega x \\
& T_{\omega} x=x .
\end{aligned}
$$

Karena $T_{\omega} x=x$, maka terbukti $x \in F\left(T_{\omega}\right)$.

Dari (i) dan (ii), dapat disimpulkan $F\left(T_{\omega}\right)=F(T)$.

Lema 2.1 dan Lema 2.2 digunakan dalam pembuktian Teorema 2.3, yang merupakan bahasan utama dalam makalah ini. Teorema tersebut mengkaji hubungan titik tetap dari $N$ pemetaan $k$-pseudononspreading sejati di ruang Hilbert.

Teorema 2.3. Asumsikan $C$ adalah himpunan bagian konveks tertutup dari ruang Hilbert riil $H$.

(a) Diberikan suatu bilangan bulat $N \geq 1$, asumsikan $T_{i}: H \rightarrow H$ adalah suatu pemetaan $k_{i}$ - pseudononspreading sejati, untuk suatu $k_{i} \in[0,1)$, $(i=1,2, \ldots, N)$. Misalkan $\left\{\lambda_{i}\right\}_{i=1}^{N}$ adalah suatu barisan positif sedemikian sehingga $\sum_{i=1}^{N} \lambda_{i}=1$. Jika $\left\{T_{i}\right\}_{i=1}^{N}$ memiliki suatu titik tetap bersama dan $\bigcap_{i=1}^{N} F\left(T_{i}\right) \neq \emptyset$, maka

$$
F\left(\sum_{i=1}^{N} \lambda_{i} T_{i}\right)=\bigcap_{i=1}^{N} F\left(T_{i}\right) .
$$


(b) Asumsikan $T_{i}: H \rightarrow H$ adalah suatu pemetaan $k_{i}$-pseudononspreading sejati untuk suatu $k_{i} \in[0,1),(i=1,2, \ldots, \mathrm{N})$. Misalkan $T_{\omega i}=\left(1-\omega_{i}\right) I+\omega_{i} T_{i}, 1 \leq$ $i \leq N$. Jika $F\left(T_{i}\right) \neq \emptyset$ maka

$$
F\left(T_{\omega 1} T_{\omega 2} \ldots T_{\omega N}\right)=\bigcap_{i=1}^{N} F\left(T_{\omega i}\right) .
$$

Bukti. Pembuktian dilakukan dengan menggunakan Induksi Matematika [3].

(a) Akan dibuktikan bahwa $F\left(\sum_{i=1}^{N} \lambda_{i} T_{i}\right)=\bigcap_{i=1}^{N} F\left(T_{i}\right)$.

(a1) Akan dibuktikan benar untuk $n=2$, yakni $\bigcap_{i=1}^{2} F\left(T_{i}\right)=F\left(\sum_{i=1}^{2} \lambda_{i} T_{i}\right)$.

Pertama-tama, akan dibuktikan bahwa $\bigcap_{i=1}^{2} F\left(T_{i}\right) \subseteq F\left(\sum_{i=1}^{2} \lambda_{i} T_{i}\right)$, dengan $0<\lambda<1$. Misalkan $x \in \bigcap_{i=1}^{2} F\left(T_{i}\right)=\left(F\left(T_{1}\right) \cap F\left(T_{2}\right)\right)$, ini berarti $x \in F\left(T_{1}\right)$ dan $x \in F\left(T_{2}\right)$. Karena $x \in F\left(T_{1}\right)$ maka $x=T_{1} x$ dan $x \in F\left(T_{2}\right)$ maka $x=T_{2} x$. Dengan perkataan lain $x=T_{1} x=T_{2} x$. Perhatikan hubungan berikut:

$$
\begin{aligned}
x & =x-\lambda T_{1} x+\lambda T_{1} x, \\
& =x-\lambda T_{1} x+\lambda T_{2} x, \\
& =T_{1} x-\lambda T_{1} x+\lambda T_{2} x, \\
& =(1-\lambda) T_{1} x+\lambda T_{2} x .
\end{aligned}
$$

Sehingga jelas bahwa $x \in F\left(\sum_{i=1}^{2} \lambda_{i} T_{i}\right)$.

Selanjutnya, akan dibuktikan bahwa $F\left(\sum_{i=1}^{2} \lambda_{i} T_{i}\right) \subseteq F\left(T_{1}\right) \cap F\left(T_{2}\right)$. Misalkan $x \in F\left(\sum_{i=1}^{2} \lambda_{i} T_{i}\right)$, tetapkan $V_{1}=I-T_{1}$ dan $V_{2}=I-T_{2}$, dengan $0<\lambda<1$. Ambil sebarang $z \in F\left(T_{1}\right) \cap F\left(T_{2}\right)$. Perhatikan bahwa

$$
\begin{aligned}
\|z-x\|^{2}= & \left\|(1-\lambda)\left(z-T_{1} x\right)+\lambda\left(z-T_{2} x\right)\right\|^{2} \\
= & (1-\lambda)\left\|z-T_{1} x\right\|^{2}+\lambda\left\|z-T_{2} x\right\|^{2}-\lambda(1-\lambda)\left\|T_{1} x-T_{2} x\right\|^{2} \\
\leq & (1-\lambda)\left(\|z-x\|^{2}+k\left\|x-T_{1} x\right\|^{2}\right)+\lambda\left(\|z-x\|^{2}+\right. \\
& \left.k\left\|x-T_{2} x\right\|^{2}\right)-\lambda(1-\lambda)\left\|T_{1} x-T_{2} x\right\|^{2} \\
= & (1-\lambda)\left(\|z-x\|^{2}+k\left\|x-T_{1} x\right\|^{2}\right)+\lambda\left(\|z-x\|^{2}+\right. \\
& \left.k\left\|x-T_{2} x\right\|^{2}\right)-\lambda(1-\lambda)\left\|\left(x-T_{1} x\right)-\left(x-T_{2} x\right)\right\|^{2} \\
= & (1-\lambda)\left(\|z-x\|^{2}+k\left\|x\left(I-T_{1}\right)\right\|^{2}\right)+\lambda\left(\|z-x\|^{2}+\right. \\
& \left.k\left\|x\left(I-T_{2}\right)\right\|^{2}\right)-\lambda(1-\lambda)\left\|x\left(I-T_{1}\right)-x\left(I-T_{2}\right)\right\|^{2} \\
= & (1-\lambda)\left(\|z-x\|^{2}+k\left\|V_{1} x\right\|^{2}\right)+\lambda\left(\|z-x\|^{2}+k\left\|V_{2} x\right\|^{2}\right)- \\
& \left.\lambda(1-\lambda) \| V_{1} x-V_{2} x\right) \|^{2} \\
= & \|z-x\|^{2}+k\left\|V_{1} x\right\|^{2}-\lambda\|z-x\|^{2}-\lambda k\left\|V_{1} x\right\|^{2}+\lambda\|z-x\|^{2}+ \\
& \left.\lambda k\left\|V_{2} x\right\|^{2}-\lambda(1-\lambda) \| V_{1} x-V_{2} x\right) \|^{2} \\
= & \left.\|z-x\|^{2}+k\left\|V_{1} x\right\|^{2}-\lambda k\left\|V_{1} x\right\|^{2}+\lambda k\left\|V_{2} x\right\|^{2}-\lambda(1-\lambda) \| V_{1} x-V_{2} x\right) \|^{2} \\
= & \|z-x\|^{2}+k\left[(1-\lambda)\left\|V_{1} x\right\|^{2}+\lambda\left\|V_{2} x\right\|^{2}\right]-\lambda(1-\lambda)\left\|V_{1} x-V_{2} x\right\|^{2},
\end{aligned}
$$

mengakibatkan

$$
\lambda(1-\lambda)\left\|V_{1} x-V_{2} x\right\|^{2} \leq k\left[(1-\lambda)\left\|V_{1} x\right\|^{2}+\lambda\left\|V_{2} x\right\|^{2}\right] .
$$


Persamaan (2.4) akan berlaku untuk suatu $k \in[0,1)$ jika $(1-\lambda) V_{1} x-$ $\lambda V_{2} x=0$, dengan menggunakan Lema 2.1(1) diperoleh

$$
(1-\lambda)\left\|V_{1} x\right\|^{2}+\lambda\left\|V_{2} x\right\|^{2}=\lambda(1-\lambda)\left\|V_{1} x-V_{2} x\right\|^{2} .
$$

Persamaan (2.4) dan persamaan (2.5) mengakibatkan:

$$
(1-k) \lambda(1-\lambda)\left\|V_{1} x-V_{2} x\right\|^{2} \leq 0
$$

karena $0<\lambda<1$ dan $k<1$, dari persamaan (2.6) diperoleh $\left\|V_{1} x-V_{2} x\right\|=$ 0 , yang mengakibatkan $T_{1} x=T_{2} x$. Karena $(1-\lambda) T_{1} x+\lambda T_{2} x=x$ maka $T_{1} x=T_{2} x=x$. Ini berarti $x \in F\left(T_{1}\right) \cap F\left(T_{2}\right)$.

(a2) Asumsikan pernyataan benar untuk $n=N-1$, sehingga

$$
\bigcap_{i=1}^{N-1} F\left(T_{i}\right)=F\left(\sum_{i=1}^{N-1} \lambda_{i} T_{i}\right)
$$

yang berarti bahwa

$$
\bigcap_{i=1}^{N-1} F\left(T_{i}\right) \subseteq F\left(\sum_{i=1}^{N-1} \lambda_{i} T_{i}\right) \operatorname{dan} F\left(\sum_{i=1}^{N-1} \lambda_{i} T_{i}\right) \subseteq \bigcap_{i=1}^{N-1} F\left(T_{i}\right) .
$$

(a3) Akan ditunjukkan pernyataan benar untuk $n=N$.

Akan dibuktikan bahwa $\bigcap_{i=1}^{N} F\left(T_{i}\right) \subseteq F\left(\sum_{i=1}^{N} \lambda_{i} T_{i}\right)$, dengan $0<\lambda<$ 1. Misal-kan $x \in \bigcap_{i=1}^{N} F\left(T_{i}\right)=\bigcap_{i=1}^{N} F\left(T_{i}\right)$, ini berarti $x \in F\left(T_{1}\right), x \in$ $F\left(T_{2}\right), \ldots, x \in F\left(T_{N}\right)$. Hal ini mengakibatkan

$$
\begin{aligned}
x \in F\left(T_{1}\right) & \Rightarrow x=T_{1} x, \\
x \in F\left(T_{2}\right) & \Rightarrow x=T_{2} x, \\
\vdots & \\
x \in F\left(T_{N}\right) & \Rightarrow x=T_{N} x .
\end{aligned}
$$

Dengan perkataan lain, $x=T_{1} x=T_{2} x=\ldots T_{N} x$. Perhatikan hubungan berikut:

$$
\begin{aligned}
x & =x-\left(\lambda_{1} T_{1} x+\lambda_{2} T_{2} x+\ldots+\lambda_{N} T_{N-1} x\right)+\left(\lambda_{1} T_{1} x+\lambda_{2} T_{2} x+\ldots+\lambda_{N-1} T_{N-1} x\right) \\
& =T_{N} x-\lambda_{1} T_{1} x-\lambda_{2} T_{2} x-\ldots-\lambda_{N-1} T_{N-1} x+\left(\lambda_{1} T_{1} x+\lambda_{2} T_{2} x+\ldots+\lambda_{N-1} T_{N-1} x\right) \\
& =\left[1-\left(\lambda_{1}+\lambda_{2}+\lambda_{3}+\ldots+\lambda_{N-1}\right)\right] x+\left(\lambda_{1}+\lambda_{2}+\lambda_{3}+\ldots+\lambda_{N-1}\right) T_{N} x, \\
& =\left(\lambda_{1} T_{1}+\lambda_{2} T_{2}+\lambda_{2} T_{2}+\ldots+\lambda_{N} T_{N}\right) x .
\end{aligned}
$$

Sehingga terbukti bahwa $x \in F\left(\sum_{i=1}^{N} \lambda_{i} T_{i}\right)$.

Selanjutnya, akan dibuktikan bahwa $F\left(\sum_{i=1}^{N} \lambda_{i} T_{i}\right) \subseteq \bigcap_{i=1}^{N} F\left(T_{i}\right)$. Misalkan $x \in F\left(\sum_{i=1}^{N} \lambda_{i} T_{i}\right)$, atau dengan perkataan lain $x=\left(\lambda_{1} T_{1}+\lambda_{2} T_{2}+\ldots+\lambda_{N} T_{N}\right) x=\left(\lambda_{1} T_{1}+\lambda_{2} T_{2}+\ldots+\lambda_{N-1} T_{N-1}\right) x+\lambda_{N} T_{N}$.

Tetapkan $V_{0}=I-T_{1}$ dan $V_{N}=I-T_{2}$. Ambil sebarang $z \in \bigcap_{i=1}^{N} F\left(T_{i}\right)$. Dari (a2) diperoleh $x=\left(\lambda_{1} T_{1}+\lambda_{2} T_{2}+\ldots+\lambda_{N} T_{N}\right) x$. Misalkan

$$
T_{0}=\left(\lambda_{1} T_{1}+\lambda_{2} T_{2}+\ldots+\lambda_{N-1} T_{N-1}\right)=\left(\sum_{i=1}^{N-1} T_{i}\right) x .
$$


Maka,

$$
\begin{aligned}
\|z-x\|^{2}= & \left\|(1-\lambda)\left(z-\sum_{i=1}^{N-1} T_{i} x\right)+\lambda\left(z-T_{N} x\right)\right\|^{2} \\
= & (1-\lambda)\left\|z-T_{0} x\right\|^{2}+\lambda\left\|z-T_{N} x\right\|^{2}- \\
& \lambda(1-\lambda)\left\|T_{0} x-T_{N} x\right\|^{2} \\
\leq & (1-\lambda)\left(\|z-x\|^{2}+k\left\|x-T_{0} x\right\|^{2}\right)+\lambda\left(\|z-x\|^{2}+\right. \\
& \left.k\left\|x-T_{N} x\right\|^{2}\right)-\lambda(1-\lambda)\left\|T_{0} x-T_{N} x\right\|^{2} \\
= & (1-\lambda)\left(\|z-x\|^{2}+k\left\|x-T_{0} x\right\|^{2}\right)+\lambda\left(\|z-x\|^{2}+\right. \\
& \left.k\left\|x-T_{2} x\right\|^{N}\right)-\lambda(1-\lambda)\left\|\left(x-T_{0} x\right)-\left(x-T_{N} x\right)\right\|^{2} \\
= & (1-\lambda)\left(\|z-x\|^{2}+k\left\|x\left(I-T_{0}\right)\right\|^{2}\right)+\lambda\left(\|z-x\|^{2}+\right. \\
& \left.k\left\|x\left(I-T_{N}\right)\right\|^{2}\right)-\lambda(1-\lambda)\left\|x\left(I-T_{0}\right)-x\left(I-T_{N}\right)\right\|^{2} \\
= & (1-\lambda)\left(\|z-x\|^{2}+k\left\|V_{0} x\right\|^{2}\right)+\lambda\left(\|z-x\|^{2}+k\left\|V_{N} x\right\|^{2}\right)- \\
& \left.\lambda(1-\lambda) \| V_{1} x-V_{2} x\right) \|^{2} \\
= & \|z-x\|^{2}+k\left\|V_{0} x\right\|^{2}-\lambda\|z-x\|^{2}-\lambda k\left\|V_{N} x\right\|^{2}+ \\
& \left.\lambda\|z-x\|^{2}+\lambda k\left\|V_{N} x\right\|^{2}-\lambda(1-\lambda) \| V_{0} x-V_{N} x\right) \|^{2} \\
= & \|z-x\|^{2}+k\left\|V_{0} x\right\|^{2}-\lambda k\left\|V_{N} x\right\|^{2}+\lambda k\left\|V_{N} x\right\|^{2}- \\
& \left.\lambda(1-\lambda) \| V_{0} x-V_{N} x\right) \|^{2} \\
= & \|z-x\|^{2}+k\left[(1-\lambda)\left\|V_{0} x\right\|^{2}+\lambda\left\|V_{N} x\right\|^{2}\right]- \\
& \lambda(1-\lambda)\left\|V_{0} x-V_{N} x\right\|^{2}, \\
& \lambda(2.7)
\end{aligned}
$$

mengakibatkan

$$
\lambda(1-\lambda)\left\|V_{0} x-V_{N} x\right\|^{2} \leq k\left[(1-\lambda)\left\|V_{0} x\right\|^{2}+\lambda\left\|V_{N} x\right\|^{2}\right] .
$$

Dari persamaan (2.8) diperoleh $(1-\lambda) V_{0} x-\lambda V_{N} x=0$, dengan menggunakan Lema 2.1(1) diperoleh

$$
(1-\lambda)\left\|V_{0} x\right\|^{2}+\lambda\left\|V_{N} x\right\|^{2}=\lambda(1-\lambda)\left\|V_{0} x-V_{N} x\right\|^{2} .
$$

Persamaan (2.8) dan persamaan (2.9) mengakibatkan

$$
(1-k) \lambda(1-\lambda)\left\|V_{0} x-V_{N} x\right\|^{2} \leq 0,
$$

karena $0<\lambda<1$ dan $k<1$, dari persamaan 2.10 diperoleh $\left\|V_{0} x-V_{N} x\right\|=$ 0 , yang mengakibatkan $T_{0} x=T_{N} x$. Karena $(1-\lambda) T_{0} x+\lambda T_{N} x=x$ maka $T_{0} x=\left(\sum_{i=1}^{N-1} T_{i}\right) x=T_{N} x=x$. Ini berarti $x \in F\left(T_{1}\right) \cap F\left(T_{2}\right) \cap \ldots F\left(T_{N}\right)$.

(b) Akan dibuktikan $F\left(T_{\omega_{1}} T_{\omega_{2}} \ldots T_{\omega_{N}}\right)=\bigcap_{i=1}^{N} F\left(T_{\omega_{i}}\right)$.

(b1) Akan dibuktikan benar untuk $n=2$, yakni $F\left(T_{\omega_{1}} T_{\omega_{2}}\right)=\bigcap_{i=1}^{2} F\left(T_{\omega_{i}}\right)$, dengan $T_{\omega_{1}}=\left(1-\omega_{1}\right) I+\omega_{1} T_{1}$ dan $T_{\omega_{2}}=\left(1-\omega_{2}\right) I+\omega_{2} T_{2}, 0<k_{i}<\omega_{i}<$ $1 / 2, i=1,2$.

(i) Akan dibuktikan bahwa $\bigcap_{i=1}^{2} F\left(T_{\omega_{i}}\right) \subseteq F\left(T_{\omega_{1}} T_{\omega_{2}}\right)$. Ambil $q \in F\left(T_{\omega_{1}}\right) \cap$ $F\left(T_{\omega_{2}}\right)$, ini berarti

$$
\begin{aligned}
& q \in F\left(T_{\omega_{1}}\right) \Rightarrow q=T_{\omega_{1}} q, \text { dan } \\
& q \in F\left(T_{\omega_{2}}\right) \Rightarrow q=T_{\omega_{2}} q .
\end{aligned}
$$


Dengan perkataan lain, $q=T_{\omega_{1}} q=T_{\omega_{2}} q$, dapat juga ditulis $q=T_{\omega_{1}} T_{\omega_{2}} q=$ $T_{\omega_{1}}\left(T_{\omega_{2}} q\right)$, sehingga dapat disimpulkan $q \in F\left(T_{\omega_{1}} T_{\omega_{2}}\right)$.

(ii) Selanjutnya, akan dibuktikan $F\left(T_{\omega_{1}} T_{\omega_{2}}\right) \subseteq F\left(T_{\omega_{1}}\right) \cap F\left(T_{\omega_{2}}\right)$. Ambil $q \in F\left(T_{\omega_{1}} T_{\omega_{1}}\right)$ dengan $q=T_{\omega_{1}} T_{\omega_{2}} q$, sedemikian sehingga

$$
T_{\omega_{2}} q=q \Rightarrow T_{\omega_{1}} q=q .
$$

Oleh karena itu, cukup dengan menunjukkan $T_{\omega_{2}} q=q$, maka akan mengakibatkan $q=T_{\omega_{1}} q$ atau $q \in F\left(T_{\omega_{1}}\right)$, sehingga $q \in F\left(T_{\omega_{1}}\right) \cap F\left(T_{\omega_{2}}\right)$. Dari Lema 2.2, diketahui bahwa $F\left(T_{\omega_{1}}\right) \cap F\left(T_{\omega_{2}}\right)=F\left(T_{1}\right) \cap F\left(T_{2}\right) \neq \emptyset$. Misalkan $p \in F\left(T_{\omega_{1}}\right) \cap F\left(T_{\omega_{2}}\right)$, maka

$$
\begin{aligned}
\|p-q\|^{2}= & \left\|p-T_{\omega_{1}} T_{\omega_{2}} q\right\|^{2} \\
= & \left\|p-\left[\left(1-\omega_{1}\right)\left(T_{\omega_{2}} q\right)+\omega_{1} T_{1} T_{\omega_{2}}\right]\right\|^{2} \\
= & \left\|\left(1-\omega_{1}\right)\left(p-T_{\omega_{2}} q\right)+\omega_{1}\left(p-T_{1} T_{\omega_{2}} q\right)\right\|^{2} \\
= & \left(1-\omega_{1}\right)\left\|p-T_{\omega_{2}} q\right\|^{2}+\omega_{1}\left\|p-T_{1} T_{\omega_{2}} q\right\|^{2}-\omega_{1}\left(1-\omega_{1}\right)\left\|T_{\omega_{2}} q-T_{1} T_{\omega_{2}} q\right\|^{2} \\
\leq & \left(1-\omega_{1}\right)\left\|p-T_{\omega_{2}} q\right\|^{2}-\omega_{1}\left(1-\omega_{1}\right)\left\|T_{\omega_{2}} q-T_{1} T_{\omega_{2}} q\right\|^{2}+ \\
& \omega_{1}\left[\left\|p-T_{\omega_{2}} q\right\|^{2}+k_{1}\left\|T_{\omega_{2}} q-T_{1} T_{\omega_{2}} q\right\|^{2}+2\left\langle p-T_{1} T_{\omega_{2}} p, T_{\omega_{2}} q-T_{1} T_{\omega_{2}} q\right\rangle\right] \\
= & \left(1-\omega_{1}\right)\left\|p-T_{\omega_{2}} q\right\|^{2}-\omega_{1}\left(1-\omega_{1}\right)\left\|T_{\omega_{2}} q-T_{1} T_{\omega_{2}} q\right\|^{2}+\omega_{1}\left[\left\|p-T_{\omega_{2}} q\right\|^{2}+\right. \\
& \left.k_{1}\left\|T_{\omega_{2}} q-T_{1} T_{\omega_{2}} q\right\|^{2}\right], \\
\leq & \left\|p-T_{\omega_{2}} q\right\|^{2}-\omega_{1}\left(1-\omega_{1}-k_{1}\right)\left\|T_{\omega_{2}} q-T_{1} T_{\omega_{2}} q\right\|^{2} \\
\leq & \|p-q\|^{2}-\omega_{1}\left(1-\omega_{1}-k_{1}\right)\left\|T_{\omega_{2}} q-T_{1} T_{\omega_{2}} q\right\|^{2} .
\end{aligned}
$$

Karena $0<k_{1}<\omega_{1}<1 / 2$, maka diperoleh

$$
\left\|T_{\omega_{2}} q-T_{1} T_{\omega_{2}} q\right\|^{2} \leq 0 .
$$

Berdasarkan definisi norm (nilai norm adalah nonnegatif), maka $\| T_{\omega_{2}} q-$ $T_{1} T_{\omega_{2}} q \|=0$, yang berarti $T_{\omega_{2}} q=T_{1} T_{\omega_{2}} q$, ini berarti

$$
T_{\omega_{2}} q \in F\left(T_{1}\right)=F\left(T_{\omega_{1}}\right),
$$

dengan kata lain $T_{\omega_{2}} q=T_{\omega_{1}} T_{\omega_{2}} q=q$ Sehingga $q \in F\left(T_{\omega_{2}}\right)$. Dapat disimpulkan bahwa $q \in F\left(T_{\omega_{2}}\right)$, ini berarti $q \in F\left(T_{\omega_{1}}\right) \cap F\left(T_{\omega_{2}}\right)$.

Dari (i) dan (ii), dapat disimpulkan bahwa $F\left(T_{\omega_{1}} T_{\omega_{2}}\right) \subset F\left(T_{\omega_{1}}\right) \cap F\left(T_{\omega_{2}}\right)$.

(b2) Asumsikan benar untuk $n=N-1$, yang berarti

$$
F\left(T_{\omega_{1}} T_{\omega_{2}} \ldots T_{\omega_{N}}\right) \subseteq \bigcap_{i=1}^{N} F\left(T_{\omega_{i}}\right) \operatorname{dan} \bigcap_{i=1}^{N} F\left(T_{\omega_{i}}\right) \subseteq F\left(T_{\omega_{1}} T_{\omega_{2}} \ldots T_{\omega_{N}}\right) .
$$

(b3) Akan ditunjukkan benar untuk $n=N$, yakni

$$
F\left(T_{\omega_{1}} T_{\omega_{2}} \ldots T_{\omega_{N}}\right)=\bigcap_{i=1}^{N} F\left(T_{\omega_{i}}\right)
$$

(i) Akan dibuktikan bahwa $\bigcap_{i=1}^{N} F\left(T_{\omega_{i}}\right) \subseteq F\left(T_{\omega_{1}} T_{\omega_{2}} \ldots T_{\omega_{N}}\right)$. Misalkan $q \in \bigcap_{i=1}^{N} F\left(T_{\omega_{i}}\right)$, ini berarti

$$
\begin{aligned}
& q \in F\left(T_{\omega_{1}}\right) \Rightarrow q=T_{\omega_{1}} q, \\
& q \in F\left(T_{\omega_{2}}\right) \Rightarrow q=T_{\omega_{2}} q .
\end{aligned}
$$


Dengan perkataan lain, $q=T_{\omega_{1}} q=T_{\omega_{2}} q=\ldots=T_{\omega_{N}} q$, dapat juga ditulis $q=T_{\omega_{1}} q=T_{\omega_{1}}\left(T_{\omega_{2}} q\right)=\ldots=T_{\omega_{1}}\left(T_{\omega_{2}} \ldots T_{\omega_{N-1}}\right) T_{\omega_{N}} q$, sehingga dapat disimpulkan $q \in F\left(T_{\omega_{1}} T_{\omega_{2}} \ldots T_{\omega_{N}}\right)$.

(ii) Akan dibuktikan $F\left(T_{\omega_{1}} T_{\omega_{2}} \ldots T_{\omega_{N}}\right) \subseteq \bigcap_{i=1}^{N} F\left(T_{\omega_{i}}\right)$. Ambil $q \in$ $F\left(T_{\omega_{1}} T_{\omega_{2}} \ldots T_{\omega_{N}}\right)$, maka $q=T_{\omega_{1}} T_{\omega_{2}} \ldots T_{\omega_{N-1}} T_{\omega_{N}} q$, sedemikian sehingga

$$
T_{\omega_{N}} q=q \Rightarrow T_{\omega_{1}} T_{\omega_{2}} \ldots T_{\omega_{N-1}} q=q .
$$

Oleh karena itu, cukup dengan menunjukkan $T_{\omega_{N}} q=q$, hal ini akan mengakibatkan $q \in \bigcap_{i=1}^{N} F\left(T_{\omega_{i}}\right)$. Dari Lema 2.2, diketahui bahwa $\bigcap_{i=1}^{N} F\left(T_{\omega_{i}}\right)=\bigcap_{i=1}^{N} F\left(T_{i}\right) \neq \emptyset$. Misalkan $p \in \bigcap_{i=1}^{N} F\left(T_{\omega_{i}}\right)$. Misalkan $T_{\omega_{0}}=T_{\omega_{1}} T_{\omega_{2}} \ldots T_{\omega_{N-1}}$ maka

$$
\begin{aligned}
\|p-q\|^{2}= & \left\|p-T_{\omega_{0}} T_{\omega_{N}} q\right\|^{2} \\
= & \left\|p-\left[\left(1-\omega_{0}\right)\left(T_{\omega_{N}} q\right)+\omega_{0} T_{0} T_{\omega_{N}} q\right]\right\|^{2} \\
= & \left\|\left(1-\omega_{0}\right)\left(p-T_{\omega_{N}} q\right)+\omega_{0}\left(p-T_{0} T_{\omega_{N}} q\right)\right\|^{2} \\
= & \left(1-\omega_{0}\right)\left\|p-T_{\omega_{N}} q\right\|^{2}+\omega_{0}\left\|p-T_{0} T_{\omega_{N}} q\right\|^{2}-\omega_{0}\left(1-\omega_{0}\right)\left\|T_{\omega_{N}} q-T_{0} T_{\omega_{N}} q\right\|^{2} \\
\leq & \left(1-\omega_{0}\right)\left\|p-T_{\omega_{N}} q\right\|^{2}-\omega_{1}\left(1-\omega_{0}\right)\left\|T_{\omega_{N}} q-T_{0} T_{\omega_{N}} q\right\|^{2}+ \\
& \omega_{1}\left[\left\|p-T_{\omega_{N}} q\right\|^{2}+k_{1}\left\|T_{\omega_{2}} q-T_{0} T_{\omega_{N}} q\right\|^{2}+2\left\langle p-T_{0} T_{\omega_{N}} p, T_{\omega_{N}} q-T_{0} T_{\omega_{N}} q\right\rangle\right] \\
= & \left(1-\omega_{0}\right)\left\|p-T_{\omega_{0}} q\right\|^{2}-\omega_{0}\left(1-\omega_{0}\right)\left\|T_{\omega_{N}} q-T_{0} T_{\omega_{N}} q\right\|^{2}+ \\
& \omega_{0}\left[\left\|p-T_{\omega_{N}} q\right\|^{2}+k_{0}\left\|T_{\omega_{N}} q-T_{0} T_{\omega_{2}} q\right\|^{2}\right], \\
\leq & \left\|p-T_{\omega_{N}} q\right\|^{2}-\omega_{0}\left(1-\omega_{0}-k_{0}\right)\left\|T_{\omega_{N}} q-T_{0} T_{\omega_{N}} q\right\|^{2} \\
\leq & \|p-q\|^{2}-\omega_{0}\left(1-\omega_{0}-k_{0}\right)\left\|T_{\omega_{N}} q-T_{0} T_{\omega_{N}} q\right\|^{2} .
\end{aligned}
$$$$
\omega_{1}\left[\left\|p-T_{\omega_{N}} q\right\|^{2}+k_{1}\left\|T_{\omega_{2}} q-T_{0} T_{\omega_{N}} q\right\|^{2}+2\left\langle p-T_{0} T_{\omega_{N}} p, T_{\omega_{N}} q-T_{0} T_{\omega_{N}} q\right\rangle\right]
$$

Karena $0<k_{0}<\omega_{0}<1 / 2$, maka diperoleh

$$
\left\|T_{\omega_{0}} q-T_{1} T_{\omega_{N}} q\right\|^{2} \leq 0
$$

Berdasarkan definisi norm, maka $\left\|T_{\omega_{2}} q-T_{1} T_{\omega_{2}} q\right\|=0$, yang berarti $T_{\omega_{N}} q=T_{0} T_{\omega_{N}} q$, ini berarti

$$
T_{\omega_{N}} q \in F\left(T_{0}\right)=F\left(T_{\omega_{0}}\right) .
$$

Dengan kata lain, $T_{\omega_{N}} q=T_{\omega_{0}} T_{\omega_{N}} q=q$. Dari (3.0.19) diperoleh $q \in$ $F\left(T_{\omega_{0}}\right)$. Ini berarti $q \in F\left(T_{\omega_{0}}\right) \cap F\left(T_{\omega_{N}}\right)$. Dari (i) dan (ii), dapat disimpulkan bahwa $F\left(T_{\omega_{1}} T_{\omega_{2}} \ldots T_{\omega_{N}}\right)=\bigcap_{i=1}^{N} F\left(T_{\omega_{i}}\right)$.

\section{Ucapan Terima kasih}

Penulis mengucapkan terima kasih kepada Bapak Dr. Syafrizal Sy, Bapak Dr. Admi Nazra, Bapak Dr. Muhafzan, Ibu Arrival Rince Putri, MT, M.Si dan Bapak Efendi, M.Si yang telah memberikan masukan dan saran sehingga makalah ini dapat diselesaikan dengan baik.

\section{Daftar Pustaka}

[1] Acedo, G.L. dan H.K. Xu. 2007. Iterative Methods for Strict Pseudo-contraction in Hilbert Spaces. Nonlinier Analysis: Theory, Methods, and Applications. 67: $2258-2271$. 
60 Desi Rahmadani

[2] Berinde, V. 2007. Iterative Approximations of Fixed Point. Verlag Berlin Heidelberg: Springer.

[3] Deng, B.C., T. Chen dan Z. Fang Li. 2012. Cyclic Iterative Method for Strictly Pseudononspreading in Hilbert Spaces. Journal Of Applied Mathematics. 2012: $1-7$.

[4] Kreyszig, E. 1978. Introductory Functional Analysis with Applications. United States of America: John Wiley and Sons. Inc

[5] Lokenath, D. dan P. Mikusinki. 1990. Introduction to Hilbert Spaces with Application. San Diego: Academic Press.

[6] Osilike, M.O. dan F.O. Isiogugu. 2011. Weak and Strong Convergence Theorems for Nonspreading-Type Mapping in Hilbert Spaces. Nonlinier Analysis: Theory, Methods, and Applications. 74: 1814 - 1822.

[7] Petrot N. dan R. Wangkere. 2011. A general iterative scheme for strict pseudononspreading mapping related to optimization problem in Hilbert Spaces. Journal of Nonlinier Analysis and Optimization. 2: 329 - 336.

[8] Ravi, P.A., D.O. Regan dan D.R. Sahu. 2009. Fixed Point Theory for Lipschitzian-type Mappings with Applications. Springer Dordrecht Heidelberg, New York.

[9] Wulandari, E.R. 1991. Theorema Titik Tetap Banach Dan Peranannya. Undergraduate thesis, FMIPA UNDIP. 